\title{
Genetics of adaptive radiation in Hawaiian species of Tetramolopium (Asteraceae). III. Evolutionary genetics of sex expression
}

\author{
RICHARD WHITKUS* $†$, HAHN DOAN $\$$ \& TIMOTHY K. LOWREY§ \\ $\dagger$ Department of Biology, Sonoma State University, Rohnert Park, CA 94928, U.S.A., $₫$ Department of Botany and \\ Plant Sciences, University of California, Riverside, CA 92521, U.S.A. and §Department of Biology, \\ University of New Mexico, Albuquerque, NM 87131, U.S.A.
}

\begin{abstract}
Despite numerous studies of speciation on oceanic islands, few insights exist on the genetic changes involved in the origin and diversification of island taxa. Here we report a genetic analysis of the evolutionary change in sex expression in Hawaiian Tetramolopium. The most diverse clade in the genus is characterized by a monoecious breeding system. The breeding system resulted from a change in sex expression in disc florets from the ancestral hermaphroditic condition to the derived male state. Analysis of an $F_{2}$ population from a cross between the two forms of sex expression indicates two regions of the Tetramolopium linkage map are associated with the loss of female function in disc florets. Quantitative trait locus mapping of the two linkage groups confirms that two loci control $56 \%$ of the phenotypic variation of the trait in the $\mathrm{F}_{2}$ population. Additive and dominance effects are apparent but no statistical evidence of epistasis was found. Several related reproductive traits also have few genetic associations on the linkage map, but are generally distinct from the control of sex expression. Although modifier loci are likely to be involved, the apparent simple genetic change underlying sex expression parallels a major evolutionary diversification in Hawaiian Tetramolopium and may have initiated the divergence of this novel clade.
\end{abstract}

Keywords: adaptive radiation, breeding system, QTL, sex expression, Tetramolopium.

\section{Introduction}

Speciation on oceanic islands is often characterized by profound divergence in morphological traits and habitat adaptations (Carlquist, 1974; Crawford et al., 1987). Within a single monophyletic lineage, this pattern of evolution is termed adaptive radiation (Carlquist, 1974) and is illustrated by many of the classic examples of insular speciation (Hawaiian examples of Silverswords, Honeycreepers, Drosophilidae, etc.). Phylogenetic analyses have provided detailed patterns of relationships among the species in island groups and genetic diversity studies have also shown that the pace of evolution has been both recent and rapid (Crawford et al., 1987). However, at the present time we have remarkably little insight into the genetic changes involved in the origin and diversification of island taxa. In plants, the advent of molecular mapping and the genetic analysis of traitcontrolling loci (Lander \& Botstein, 1989), combined with the ability to obtain fertile hybrids from many

*Correspondence. E-mail: whitkus@sonoma.edu interspecific crosses, allows analysis of the genetic basis of traits involved in the origin of both individual species as well as entire lineages.

A model system for investigating insular adaptive radiation exists in Hawaiian Tetramolopium, a monophyletic group of 11 species (Lowrey, 1986). The group is comprised of three descendent lineages (clades) that are differentiated by a combination of morphological traits and adaptations to different ecological zones. The most diverse clade in the genus, the taxonomic section Tetramolopium (seven species), is characterized by nine morphological traits, the majority of which are related to reproduction (Lowrey, 1986; Fig. 1). The most distinctive feature of this clade is its sex expression (distribution of sexes within and among individuals). The clade consists entirely of monoecious (female and male flowers) species and is derived from a lineage of gynomonoecious (female and hermaphroditic flowers) species. The change in sex expression in section Tetramolopium correlates with the largest amount of genetic variation and morphological diversity in the Hawaiian group (Lowrey \& Crawford, 1985; Lowrey, 1986; Okada 

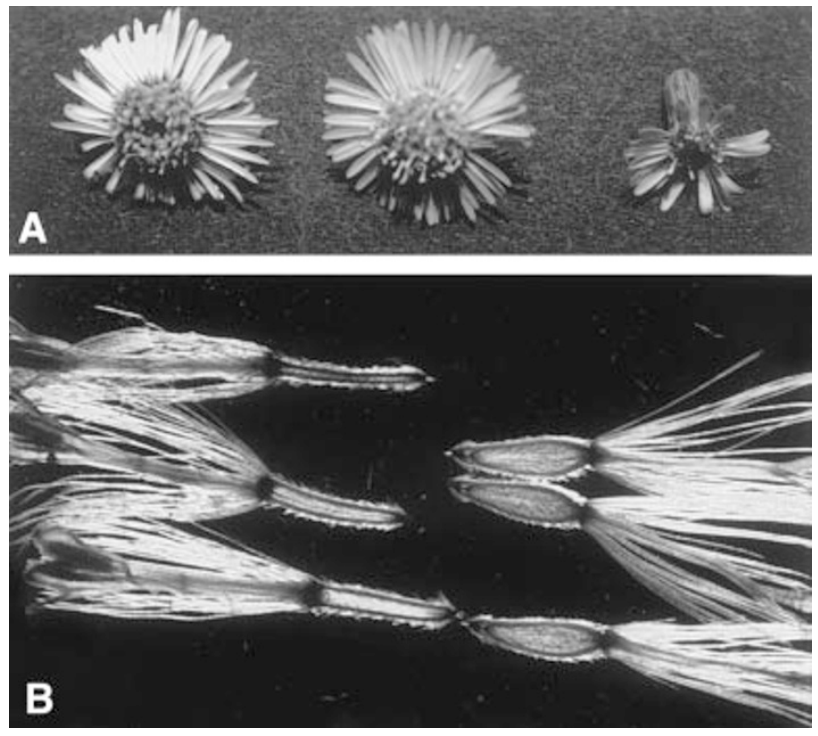

Fig. 1 Trait differences in Hawaiian Tetramolopium as represented by (A) capitulum size and floret (flower) number in Tetramolopium rockii (left), T. humile (right) and the $\mathrm{F}_{1}$ hybrid (middle) (1.5× magnification) and (B) disc floret female-sterile (left, linear ovary) and female-fertile (right, fusiform ovary). Disc florets on the left are restricted to $T$. rockii and the $\mathrm{F}_{1}$ hybrid whereas those on the right are restricted to $T$. humile, although this photo is from an $F_{2}$ individual containing both ( $5 \times$ magnification).

et al., 1997), adaptation to low elevation, xeric habitats (Lowrey, 1986, 1995), and the derivation of a species that originated after long-distance dispersal to the Cook Islands of the south Pacific (Lowrey, 1995). This combination of features indicates that the change in sex expression has paralleled a major evolutionary shift in the genus. In this report we demonstrate that this switch is mainly under simple genetic control in Hawaiian Tetramolopium.

\section{Materials and methods}

All Hawaiian species of Tetramolopium are easily grown in the glasshouse with no effect on trait expression (high heritabilities). Artificial hybridizations resulting in fertile hybrids are also easily produced between all species (Lowrey, 1986). Our analysis focused on a cross between species from two clades. The ancestral clade in Hawaii is section Alpinum, represented by a single, gynomonoecious species ( $T$. humile) found in the alpine zone on the big island of Hawaii and on Maui. Section Tetramolopium is derived and represented in the analysis by $T$. rockii, a species that occurs on lithified calcareous dunes on the northern side of the island of Molokai. Source of seeds, plant cultivation, and cross hybridization procedures have been described previously (Whitkus, 1998).
A single $F_{2}$ population of 342 individuals provided the material for subsequent analyses with the number of individuals for each analysis determined by the available data. Seven traits (number of capitula [primary inflorescences] per flowering shoot, capitulum width, number of ray florets, number of disc florets, number of femalefertile disc florets, percentage female-fertile disc florets, ray/disc floret ratio) which characterize section Tetramolopium were recorded on all 342 individuals. The number of capitula per plant and capitulum width were recorded from greenhouse grown plants at first flowering. Capitula were collected and dissected to count the number of ray and disc florets. Disc florets from each capitulum were placed in a glycerol:water (1:1) solution to clear the ovary, followed by recording the number of female-fertile disc florets using a dissecting microscope (Fig. 1b). The traits percentage female-fertile disc florets and ray/disc ratio were derived from the measured traits. The level of sex expression was measured by the percentage female-fertile disc florets, where monoecy is a value of 0 ( $T$. rockii) and gynomonoecy a value of 100 (T. humile). This trait has a U-shaped distribution in the $\mathrm{F}_{2}$ which could not be transformed to approximate a normal distribution. The chi-squared and permutation tests employed in this study do not rely on normally distributed data (Churchill \& Doerge, 1994) and simulations have shown that non-normal data can be treated as continuous data in quantitative trait locus (QTL) analysis without significant loss of efficiency in QTL determination (Visscher et al., 1996).

The distribution of percentage female-fertile disc florets in the $342 \mathrm{~F}_{2}$ individuals was fitted to Mendelian models of 1-4 loci (Table 1). Each model was tested with no interaction, the same as a mono-, di-, trior tetrahybrid cross for 1, 2, 3 or 4 genes, respectively. Interactions among loci were also tested. The one-gene model analysed interactions among alleles via dominance. In multiple-gene models the interactions tested were complementation, where a phenotype is produced by the contribution of at least one dominant allele from each locus; dominant suppression, where a phenotype is suppressed by a dominant allele from a locus; and dominant or recessive epistasis from one locus. In the three-gene model, complementation was tested using both three and two genes, and dominant suppression with both one and two genes. In the four-gene model, complementation was tested with four, three and two genes, and dominant suppression with one, two and three genes. All the tests represent extensions of Mendelian analyses found in standard textbooks (e.g. Griffiths et al., 1996). The significance value $(P<0.05)$ to reject a model was determined using a chi-squared test and adjusted by sequential Bonferroni correction (Rice, 1989). 
Table 1 Results of Mendelian analyses for percentage female-fertile disc florets in Hawaiian Tetramolopium

\begin{tabular}{llrr}
\hline Gene model & Interaction (no. of genes) & \multicolumn{1}{c}{$P$} & d.f. \\
\hline 1 & None & $<0.001$ & 2 \\
& Dominance & $<0.001$ & 1 \\
& None & $<0.001$ & 3 \\
& Complementation & 0.347 & 1 \\
& Dominant suppression & $<0.001$ & 1 \\
Recessive epistasis & 0.016 & 2 \\
Dominant epistasis & $<0.001$ & 2 \\
None & $<0.001$ & 7 \\
3 & Complementation (3) & $<0.001$ & 1 \\
Complementation (2) & $<0.001$ & 1 \\
Dominant suppression (1) & $<0.001$ & 1 \\
& Dominant suppression (2) & 0.719 & 1 \\
& Recessive epistasis (1) & $<0.001$ & 4 \\
Dominant epistasis (1) & $<0.001$ & 2 \\
None & $<0.001$ & 12 \\
Complementation (4) & $<0.001$ & 1 \\
4 & Complementation (3) & $<0.001$ & 1 \\
Complementation (2) & $<0.001$ & 1 \\
Dominant suppression (1) & $<0.001$ & 1 \\
Dominant suppression (2) & 0.426 & 1 \\
& Dominant suppression (3) & $<0.001$ & 1 \\
Recessive epistasis (1) & $<0.001$ & 7 \\
Dominant epistasis (1) & $\leq 0.001$ & 1 \\
\hline & & &
\end{tabular}

Subsequent analyses were facilitated by a genetic marker-based linkage map in Tetramolopium. The map consists of 125 genomic RFLP and RAPD markers mapped as 117 different loci on nine linkage groups for a total map length of 665.7 centiMorgans $(\mathrm{cM})$, covering over $65 \%$ of the Tetramolopium genome (Whitkus, 1998). A subset of the $F_{2}$ individuals scored for morphological traits in the present study were used in the production of the linkage map. This subset provided 71 individuals with both trait data and 125 marker loci genotypes. Associations between phenotypic traits and mapped marker loci were found using simple linear regression as implemented in QTL CARTOGRAPHER version 1.13 (Basten et al., 1999). The experiment(genome)-wise significant level $(P<0.05)$ was determined for each trait by 1000 permutations (Churchill \& Doerge, 1994).

Quantitative trait loci for sex expression were identified using 15 marker loci (TH237, TH240, TR21B, TR208, A04-800, A04-1100, B04-CD, B04-2100, B08800, B08-980, B08-1100, C07-600, E02-950, E19-1000, K11-1150; locus names follow Whitkus, 1998) on the two linkage groups that showed significant association with percentage female-fertile disc florets in the linear regression analysis. Genotypes were obtained from 215 individuals in the $\mathrm{F}_{2}$ population. Plant genomic DNA, restriction fragment length polymorphism, and random amplified polymorphic DNA genetic markers were prepared by previously described methods (Okada et al., 1997; Whitkus, 1998). QTL analysis was performed with MAPMAKER/QTL 1.1 (Paterson et al., 1988). Initial scans of the two linkage groups provided putative locations for two QTLs. Increased sensitivity to locating QTL position was accomplished in subsequent scans, with each scan allowing one QTL, identified in the first scan, to explain the genetic component of the trait on one linkage group and scanning the other linkage group. No additional QTLs were observed in these analyses or in the final scan where both QTLs were allowed to control the genetic component of the trait. Confirmation of the number and locations of the QTLs on the two linkage groups was provided by 1000 permutations of the data with QTL CARTOGRAPHER. The permutation test determined a logarithm of the odds ratio (LOD) equal to or greater than 12.1 as the experiment-wise significance level for declaring a single QTL.

Mean phenotypic trait values were determined for nine possible two-locus genotypic classes at molecular marker loci closely linked to the identified QTLs (B04CD and TR182). Evidence for two-locus epistasis was examined by looking for a significant interaction term in a parametric two-factor analysis of variance (Dixon, 1990).

\section{Results}

The $F_{1}$ hybrid plants displayed character dominance towards section Tetramolopium for all seven traits (e.g. Fig. 1a). In the Mendelian analysis of $342 F_{2}$ individuals, the percentage of disc florets that are female-fertile (Fig. 1b) fitted four of the 23 models: complementation and recessive epistasis (two-gene models), and dominant suppression via two genes in both the three- and four-gene models (Table 1). All other models and forms of interaction failed to fit Mendelian expectations $\left(P<0.001 ; P_{0.05}\right.$ adjusted by sequential Bonferroni correction).

Linear regression revealed that five marker loci on the Tetramolopium linkage map exhibit significant association with percentage female-fertile disc florets (experiment-wise $F=13.122$ for alpha of 0.05 ). The marker loci are restricted to two of the nine linkage groups (C and D, Fig. 2) and occur within a 7-cM region on linkage group $\mathrm{C}$ and a $22-\mathrm{cM}$ region on linkage group D. Close linkage among the loci on each linkage group agreed with the Mendelian analysis of two trait-controlling loci. One other trait associated with sex expression (total number of female-fertile disc florets) shared a similar distribution of genetic correlations and reflects the high phenotypic correlation (0.91, product-moment 
correlation) between these two traits. The remaining traits do not share a common major genetic component with sex expression (Fig. 2). This analysis shows the whole sex expression syndrome is composed of several independent traits with their major controlling loci spread over the Tetramolopium genome.

QTL mapping on linkage groups $\mathrm{C}$ and D revealed one region on each linkage group that supported a QTL for percentage female-fertile disc florets (Fig. 2). Both putative QTLs exhibit a strong additive component. The presence of a $T$. humile allele on linkage groups $\mathrm{C}$ and $\mathrm{D}$ increased the percentage of female-fertile disc florets by values of 26.2 and 31.3, respectively. The highest LOD for declaring QTLs was obtained with an unconstrained two-QTL model (both dominance and additive effects

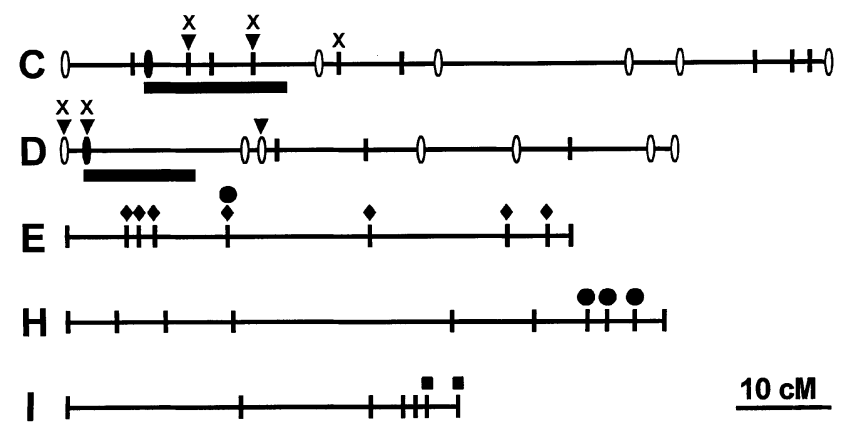

Fig. 2 Linkage groups in Hawaiian Tetramolopium $(n=9)$ with significant associations $(P<0.05)$ between marker loci (vertical ticks and ellipses) and traits associated with sex expression (symbols). These sex-expression traits are: percentage female-fertile disc florets $(\boldsymbol{\nabla})$; number of female-fertile disc florets $(\mathrm{X})$; number of disc florets $(\mathbf{\square})$; number of ray florets $(\bullet)$; and ray to disc ratio $(\bullet)$. No significant association exists for number of capitula or capitulum width. Vertical ellipses are marker loci genotyped for QTL analysis, and horizontal bars are positions of likely QTLs \pm 1 LOD. Letters are linkage group designations from the Tetramolopium map (Whitkus, 1998). Solid ellipses on linkage groups C and D are marker loci used in test of epistasis. for each locus, $L O D=26.78)$. Slightly decreased LODs were obtained when the two-QTL model allowed additive or dominance effects on linkage group $\mathrm{C}$ and additive effects on linkage group D (LODs 25.37 to 26.37). All other two-QTL models had a significant reduction in the LOD score (falling below 22.0). The two-QTL model explained $56 \%$ of the phenotypic variance of percentage female-fertile disc florets in the $\mathrm{F}_{2}$. As independent loci, the phenotypic variance explained by the QTL on linkage group C was $30 \%$ and the QTL on linkage group D explained 33\%.

Two codominant molecular marker loci were closest to the identified QTLs. On linkage group $\mathrm{C}$ the marker locus (B04-CD) was $4 \mathrm{cM}$ from the peak LOD of the putative QTL, and on linkage group D the marker locus (TR182) was $12 \mathrm{cM}$ from the peak LOD of the putative QTL. Mean trait values for percentage female-fertile disc florets in the nine genotypic classes of these two markers are presented in Table 2. As found in the QTL analysis, addition of $T$. humile alleles at either molecular marker locus resulted in an increase in percentage female-fertile disc florets. No evidence of epistatic interaction was found as the interaction term of the two-factor analysis of variance was not significant $(P>0.95)$. This result is at variance with the Mendelian analysis. The Mendelian analysis used a larger population of individuals and did not rely on linked markers to estimate effects of each trait locus. Considering both analyses, epistatic interaction among traitcontrolling loci for sex expression in Tetramolopium is suggested but equivocal.

\section{Discussion}

Comparative studies show that variation in the expression of sex in flowering plants represents a continuum. At one end of this continuum, species with initially hermaphroditic flowers may selectively abort the development of female (gynoecium) or male (androecium) organs, whereas the other extreme is sterility where male

Table 2 Mean trait values (number of individuals, standard error of the mean) for percentage female-fertile disc florets in Tetramolopium over nine genotypic classes of molecular markers on linkage groups C (B04-CD) and D (TR182) with closest linkage to putative QTLs. Allele $A$ is from $T$. rockii (monoecious) and allele $B$ from $T$ humile (gynomonoecious)

\begin{tabular}{lrcc}
\hline & \multicolumn{3}{c}{ B04-CD } \\
\cline { 2 - 4 } TR182 & \multicolumn{1}{c}{$A A$} & $A B$ & \multicolumn{1}{c}{$B B$} \\
\hline$A A$ & $5.14(28,3.14)$ & $33.71(21,6.19)$ & $32.00(2,0.0)$ \\
$A B$ & $21.50(50,3.87)$ & $53.13(64,4.10)$ & $65.00(8,13.04)$ \\
$B B$ & $50.38(13,8.04)$ & $76.90(20,6.06)$ & $90.40(5,8.41)$ \\
\hline
\end{tabular}


or female gametophytes fail to differentiate (Irish \& Nelson, 1989; Dellaporta \& Calderon-Urrea, 1993; Grant et al., 1994). Among related species, sex expression evolution typically involves the loss of one of the sex functions through a developmental switch following floral induction (Charlesworth \& Charlesworth, 1978; Irish \& Nelson, 1989; Grant et al., 1994; Baker et al., 1997). Although the literature on the control of sex expression indicates that a diverse set of mechanisms determines sex in a flower, the genotype represents the primary cause (Irish \& Nelson, 1989; Dellaporta \& Calderon-Urrea, 1993; Grant et al., 1994).

In male disc florets of monoecious species of Tetramolopium the ovary must develop as it represents the base of the flower upon which the petals and stamens are borne. The style still functions to push pollen out of the tube formed by the fused stamens, the typical method of pollen presentation in the Asteraceae. Therefore, derivation of a clade of monoecious species from gynomonoecious species involved the loss of female function (formation of the ovule) in the gynoecium. Our analysis shows that two major genes are involved in this sterilization. Clearly, modifier genes are involved in the present study because approximately $40 \%$ of the phenotypic variation for percentage female-fertile disc florets remains unaccounted in the analysis. Other QTL studies of trait differences between species typically show that genes of major effect are accompanied by modifiers (Bernacchi \& Tanksley, 1997; Bradshaw et al., 1998). Detailed QTL analysis, now in progress, should reveal greater detail on the genetic architecture of the sex expression syndrome in Tetramolopium.

Lowrey (1986) recognized a suite of associated character changes in Hawaiian Tetramolopium with the switch in sex expression. A change in plant growth habit, the presence of storage parenchyma in leaves, and six reproductive traits (number of capitula, capitulum width, number of disc florets, disc floret colour, number of ray florets, and ray floret length) represent the sex expression syndrome in Tetramolopium (Lowrey, 1986). The strong association of these traits within section Tetramolopium suggests a common genetic component. Our finding that linkage groups C, D, and E exhibit an association with two traits argues for pleiotropic control acting to produce part of the sex expression syndrome, but the current evidence is not strong. The large phenotypic correlation between number of female-fertile disc florets and percentage female-fertile disc florets suggests that these traits may represent one common trait. It is interesting to note, however, that these traits express smaller and different phenotypic correlation with the total number of disc florets $(0.26$ for number of female-fertile disc florets, -0.06 for percentage fertile disc florets). Thus control of the development of number of disc florets appears unrelated to sex expression, as evidenced by control for total number of disc florets occurring on a separate linkage group (I in Fig. 2). Epistatic interaction for the two QTLs found for percentage female-fertile disc florets is also not significant. Given the small mapping population for the other traits measured in this study, these results are tentative. However, the suite of traits seen in the change in sex expression in Tetramolopium does not have an obvious simple genetic basis. Instead, several regions of the genome are involved suggesting several independently controlled traits make up the sex expression syndrome in Hawaiian Tetramolopium.

Lowrey $(1986,1995)$ also noted that section Tetramolopium occurs in more xeric habitats than other members of the genus. This shift from mesic to xeric habitats along with a change in breeding system has been noted in unrelated groups of flowering plants (Hart, 1985; Barrett, 1992; Weller et al., 1995). No empirical data exist which explain the shift in habitat, nor is the correlation perfect because hermaphroditic species related to monoecious or dioecious species also occur in xeric habitats.

It is becoming evident that major morphological changes in plants can be under fairly simple genetic control (Bachmann, 1983; Gottlieb, 1984) especially via transcriptional regulation (Doebley \& Lukens, 1998). In this context, the current study supports models of breeding system evolution (Charlesworth \& Charlesworth, 1978), or novel adaptation (Orr \& Coyne, 1992) in which few genes are required to account for major evolutionary changes. The simple genetic basis for sex expression in Tetramolopium correlates with the diversification of this novel clade. Whether this genetic change stimulated the diversification or was only one of a suite of traits that were an outcome of this diversification remains an intriguing, unanswered question.

The elucidation of the genetic architecture of sex expression in Tetramolopium has implications beyond understanding evolution in the insular genus. Sex expression evolution is a major source of diversification at the specific and generic level in the Asteraceae, with approximately 1535 genera and 23000 species presently recognized in the family (Bremer, 1994). The switch between female fertility and sterility responsible for the change from gynomonoecy to monoecy in Tetramolopium is particularly common in the Asteraceae and is an important character for taxonomic delimitation in the family (Bremer, 1994). The explanation of the genetic control of sex expression in Tetramolopium is one step towards developing a general model for the genetics of sex expression in one of the largest and most diverse flowering plant families. 


\section{Acknowledgements}

We thank V. Weng for laboratory assistance, D. Gessler for technical advice, and E. Charnov, N. Ellstrand, $\mathrm{S}$. $\mathrm{Xu}$, and two anonymous reviewers for comments on an early version of the manuscript. Support for this work was provided by NSF grants (DEB-9204261 to R.W. and DEB-9200578 to T.K.L.) and the UC Riverside Agricultural Experimental Station funds to R.W.

\section{References}

BACHMANN, K. 1983. Evolutionary genetics and the genetic control of morphogenesis in flowering plants. In: Hecht, M. K., Wallace, B. and Ghillean, T. P. (eds) Evolutionary Biology, vol. 16, pp. 157-208. Plenum Press, New York.

BAKER, S. C., ROBINSON-BEERS, K., VILLANUEVA, J. M., GAISER, J.C. AND GASSER, C. S. 1997. Interactions among genes regulating ovule development in Arabidopsis thaliana. Genetics, 145, 1109-1124.

BARRETT, S. C. 1992. Gender variation and the evolution of dioecy in Wurmbea dioica (Liliaceae). J. Evol. Biol., 5, 423-444.

BASTEN, C. J., WEIR, B. S. AND ZENG, Z.-B. 1999. QTL CARTOGRAPHER, version 1.13. Department of Statistics, North Carolina State University, Raleigh, NC.

BERNACCHI, D. AND TANKSLEY, S. D. 1997. An interspecific backcross of Lycopersicon esculentum $\times$ L. hirsutum: linkage analysis and a QTL study of sexual compatibility factors and floral traits. Genetics, 147, 861-877.

BRADSHAW, H. D., JR., OTTO, K. G., FREWEN, B. E., McKAY, J. K. AND SCHEMSKE, D. W. 1998. Quantitative trait loci affecting differences in floral morphology between two species of monkeyflower (Mimulus). Genetics, 149, 367-382.

BREMER, K. 1994. Asteraceae: Cladistics and Classification. Timber Press, Portland, OR.

CARlQuist, s. 1974. Island Biology. Columbia University Press, New York.

CHARlesworth, B. AND CHARlesworth, D. 1978. Population genetics of partial male-sterility and the evolution of monoecy and dioecy. Heredity, 41, 137-153.

CHURChill, G. A. AND DOERGE, R. W. 1994. Empirical threshold values for quantitative trait mapping. Genetics, 138, 963-971.

CRAWFORD, D. J., WHITKUS, R. AND STUESSY, T. F. 1987. Plant evolution and speciation on oceanic islands. In: Urbanska, K. M. (ed.) Differentiation Patterns in Higher Plants, pp. 183-199. Academic Press, London.

DEllaporta, S. L. AND CALDERon-UrReA, A. 1993. Sex determination in flowering plants. Pl. Cell, 5, 1241-1251.

DIXON, w. J. (ed.) 1990. BMDP Statistical Software. University of California Press, Berkeley.
DOEBLEY, J. AND LUKENS, L. 1998. Transcriptional regulators and the evolution of plant form. Pl. Cell, 10, 1075-1082.

GOTTLieB, L. D. 1984. Genetics and morphological evolution in plants. Am. Nat., 123, 681-709.

GRANT, S., HOUBEN, A., VYSKOT, B., SIROKY, J., PAN, W.-H., MACAS, J. AND SADLER., H. 1994. Genetics of sex determination in flowering plants. Dev. Gen., 15, 214-230.

GRIFFITHS, A. J. F., MILLER, J. H., SUZUKI, D. T., LEWONTIN, R. C. AND GElbart, w. M. 1996. An Introduction to Genetic Analysis, 6th edn. W. H. Freeman, New York.

HART, J. A. 1985. Evolution of dioecism in Lepechinia Willd. sect. Parviflorae (Lamiaceae). Syst. Bot., 10, 147-154.

IRISH, E. E. AND NELSON, T. 1989. Sex determination in monoecious and dioecious plants. Pl. Cell, 1, 737-744.

LANDER, E. S. AND BOTSTEIN, D. 1989. Mapping Mendelian factors underlying quantitative traits using RFLP linkage maps. Genetics, 121, 185-199.

LOWREY, T. K. 1986. A biosystematic revision of Hawaiian Tetramolopium (Compositae: Astereae). Allertonia, 4, 203265.

LOWREY, T. K. 1995. Phylogeny, adaptive radiation, and biogeography of Hawaiian Tetramolopium (Asteraceae: Astereae). In: Wagner, W. L. and Funk, V. A. (eds) Hawaiian Biogeography, pp. 195-220. Smithsonian Institution Press, Washington, DC.

LOWREY, T. K. AND CRAWFORD, D. J. 1985. Allozyme divergence and evolution in Tetramolopium (Compositae: Astereae) on the Hawaiian Islands. Syst. Bot., 10, 64-72.

OKADA, M., WhitKUS, R. AND LOWREY, T. K. 1997. Genetics of adaptive radiation in Hawaiian and Cook Islands species of Tetramolopium (Asteraceae; Astereae). I. Nuclear RFLP marker diversity. Am. J. Bot., 84, 1236-1246.

ORR, H. A. AND COYNE, J. A. 1992. The genetics of adaptation: a reassessment. Am. Nat., 140, 725-742.

PATERSON, A., LANDER, E. S., HeWITT, J. D., PETERSON, S., LINCOLN, S. E. AND TANKSLEY, S. D. 1988. Resolution of quantitative traits into Mendelian factors by using a complete linkage map of restriction fragment length polymorphisms. Nature, 335, 721-726.

RICE, W. R. 1989. Analyzing tables of statistical tests. Evolution, 43, 223-225.

VISSCHER, P. M., HALEY, C. S. AND KNOTT, S. A. 1996. Mapping QTLs for binary traits in backcross and $F_{2}$ populations. Genet. Res., 68, 55-63.

WELler, S. G., WAGNER, W. L. AND SAKAI, A. K. 1995. A phylogenetic analysis of Schiedea and Alsinidendron (Caryophyllaceae: Alsinoideae): Implication for the evolution of breeding systems. Syst. Bot., 20, 315-337.

WHITKUS, R. 1998. Genetics of adaptive radiation in Hawaiian and Cook Islands species of Tetramolopium (Asteraceae). II. Genetic linkage map and its implications for interspecific breeding barriers. Genetics, 150, 1209-1216. 